\title{
Dissipative Spherical Gravitational Collapse of Isotropic Fluid
}

\author{
B. C. Tewari, Kali Charan \\ Department of Mathematics, Kumaun University, S.S.J. Campus, Almora, India \\ Email: drbctewari@yahoo.co.in, kcyadav2008@gmail.com
}

Received 31 January 2015; accepted 22 March 2015; published 25 March 2015

Copyright (C) 2015 by authors and Scientific Research Publishing Inc.

This work is licensed under the Creative Commons Attribution International License (CC BY). http://creativecommons.org/licenses/by/4.0/

(c) (i) Open Access

\begin{abstract}
We present a number of parametric class of exact solutions of a radiating star and the matching conditions required for the description of physically meaningful fluid. A number of previously known class of solutions have been rediscovered which describe well behaved nature of fluid distributions. The interior matter fluid is shear-free spherically symmetric isotropic and undergoing radial heat flow. The interior metric obeyed all the relevant physical and thermodynamic conditions and matched with Vaidya exterior metric over the boundary. Initially the interior solutions represent a static configuration of perfect fluid which then gradually starts evolving into radiating collapse. The apparent luminosity as observed by the distant observer at rest at infinity and the effective surface temperature are zero in remote past at the instant when collapse begins and at the stage when collapsing configuration reaches the horizon of the black hole.
\end{abstract}

\section{Keywords}

Exact Solutions, Radiating Star, Gravitational Collapse, Black Hole

\section{Introduction}

Modern astrophysics opens numerous challenges for researchers to study various aspects of gravitational collapse. It is one of the most interesting phenomena in relativistic astrophysics. When a body does not have substantially strong pressure gradient force, it may continue collapsing because of its own gravity. Therefore, a detailed description of gravitational collapse of massive stars and the modelling of the structure of compact objects under various conditions is the problem that attracts significant attention of researchers in relativistic astrophysics. With reference to cosmic censorship conjecture gravitational collapse must terminate into a black hole (Penrose [1]), but there are several counter examples where a naked singularity has more chances to be formed (Joshi and Malafarnia [2] and references therein). Still there is no established theory which can determine whether 
there will be the formation of a black hole or a naked singularity.

To understand the nature of collapse, it is necessary to form a realistic model, which requires solving non linear differential equations. Solving such equations is a very difficult task; various efforts have been made in this direction. The pioneering work in this area was started by Oppenheimer and Snyder [3], in which they assumed a spherically symmetric distribution of matter, adiabatic flow and the equation of state in the form of dust with Schwarzschild exterior. Later on, taking into account the outgoing radiation from collapsing spherical fluid, Vaidya [4] initiated the problem and the modified equations proposed by Misner [5] and Lindquist et al. [6] for an adiabatic distribution of matter.

It is an established fact that gravitational collapse is a highly dissipating energy process (Herrera and Santos [7], Herrera et al. [8], Mitra [9] and references therein) which plays a dominant role in the formation and evolution of stars. In diffusion approximation the dissipative model is described by the heat flow type vector. Santos [10] studied the junction conditions of collapsing spherically symmetric shear-free non-adiabatic fluid with radial heat flow which was based on relativistic models suggested by Glass [11]. On the similar ground a number of stellar models (Maiti [12], de Oliveira et al. [13], Bonnor et al. [14], Banerjee et al. [15], Herrera et al. [16], Tewari [17]-[20], Tewari and Charan [21], Sharma and Tikekar [22], Ivanov [23], Pinheiro and Chan [24] and also references therein) have been reported with the impact of various factors such as shear, inhomogeneity, anisotropy, electromagnetic field and various dissipative processes on the evolution.

Shear-free fluid distributions with radial heat flow are often studied in order to simplify the calculations and allow realistic analytic solutions. In view of the above arguments we present some special solutions of Tewari [20] and demonstrate a detailed study of one such solution in order to construct a realistic model of collapsing radiating star. The interior space-time metric is matched with Vaidya exterior metric (Vaidya [25]) over the boundary, and the final fate of our model is formation of a black hole. The paper is organised as follows. In Section 2 the field equations and the junction conditions which match the interior metric of the collapsing fluid with the exterior metric are given. In Section 3 a new class of exact solutions of the field equations and a table of some special solutions are presented. In Section 4 a detailed study of a class of solutions for a collapsing radiating star is given. Section 5 describes temperature profile of the solution and finally in Section 6 some concluding remarks have been made.

\section{Field Equations and Junction Conditions}

Space-time of a radiating star is divided by its boundary into two significant regions, the interior space-time $M^{-}$and the exterior space-time $M^{+}$for a stellar model. Each of these specific regions is described by a peculiar smooth time-like three dimensional hyper surface containing $\Sigma$ as its boundary. When approaching $\Sigma$ from the exterior or the interior space time, we require

$$
\left(\mathrm{d} s_{+}^{2}\right)_{\Sigma}=\left(\mathrm{d} s_{-}^{2}\right)_{\Sigma}=\mathrm{d} s_{\Sigma}^{2}
$$

with this the line elements match on the boundary $\Sigma$.

Spherically symmetric collapsing distribution of dissipative fluid in the form of heat flow with a boundary of a time like spherical surface $\Sigma$ with metric is considered as

$$
\mathrm{d} s_{\Sigma}^{2}=-\mathrm{d} \tau^{2}+\mathcal{R}^{2}(\tau)\left(\mathrm{d} \theta^{2}+\sin ^{2} \theta \mathrm{d} \phi^{2}\right)
$$

The metric in the interior of a shear-free spherically symmetric fluid distribution is given by

$$
\mathrm{d} s_{-}^{2}=-A^{2}(r, t) \mathrm{d} t^{2}+B^{2}(r, t)\left\{\mathrm{d} r^{2}+r^{2}\left(\mathrm{~d} \theta^{2}+\sin ^{2} \theta \mathrm{d} \phi^{2}\right)\right\}
$$

The energy-momentum tensor for the matter distribution with radial heat flow is

$$
T_{\mu \nu}=(\epsilon+p) w_{\mu} w_{\nu}+p g_{\mu \nu}+q_{\mu} w_{\nu}+q_{\nu} w_{\mu}
$$

where $\epsilon$ is the energy density of the fluid, $p$ the isotropic pressure, $w_{\mu}$ is the four-velocity and $q_{\mu}$ the radial heat flow vector. Assuming comoving coordinates, we have

$$
w^{\mu}=\frac{1}{A} \delta_{0}^{\mu}
$$

The heat flow vector $q^{\mu}$ is orthogonal to the velocity vector so that $q^{\mu} w_{\mu}=0$ and takes the form 


$$
q^{\mu}=q \delta_{1}^{\mu}
$$

The line element (3) corresponds to shear-free spherically symmetric fluid (Glass [26]) as the shear tensor vanishes identically. The fluid collapse rate $\Theta=w_{; \mu}^{\mu}$ of the fluid distribution (3) is given as

$$
\Theta=\frac{3 \dot{B}}{A B}
$$

here and hereafter the dots and primes stand respectively for differentiation with respect to $t$ and $r$.

Non-trivial Einstein's field equations in view of (3) and (4) are given by following system of equations

$$
\begin{aligned}
& \kappa \epsilon=-\frac{1}{B^{2}}\left(\frac{2 B^{\prime \prime}}{B}-\frac{B^{\prime 2}}{B^{2}}+\frac{4 B^{\prime}}{r B}\right)+\frac{3 \dot{B}^{2}}{A^{2} B^{2}} \\
& \kappa p=\frac{1}{B^{2}}\left(\frac{B^{\prime 2}}{B^{2}}+\frac{2 A^{\prime} B^{\prime}}{A B}+\frac{2 A^{\prime}}{r A}+\frac{2 B^{\prime}}{r B}\right)+\frac{1}{A^{2}}\left(-\frac{2 \ddot{B}}{B}-\frac{\dot{B}^{2}}{B^{2}}+\frac{2 \dot{A} \dot{B}}{A B}\right) \\
& \kappa p=\frac{1}{B^{2}}\left(\frac{B^{\prime \prime}}{B}-\frac{B^{\prime 2}}{B^{2}}+\frac{B^{\prime}}{r B}+\frac{A^{\prime \prime}}{A}+\frac{A^{\prime}}{r A}\right)+\frac{1}{A^{2}}\left(-\frac{2 \ddot{B}}{B}-\frac{\dot{B}^{2}}{B^{2}}+\frac{2 \dot{A} \dot{B}}{A B}\right) \\
& \kappa q=\frac{-2}{A B^{2}}\left(-\frac{\dot{B}^{\prime}}{B}+\frac{B^{\prime} \dot{B}}{B^{2}}+\frac{A^{\prime} \dot{B}}{A B}\right)
\end{aligned}
$$

the coupling constant in geometrized units is $\kappa=8 \pi \quad$ (i.e. $G=c=1$ ).

The exterior space-time is described by Vaidya's metric [25] which represents an outgoing radial flow of radiation

$$
\mathrm{d} s_{+}^{2}=-\left(1-\frac{2 M(v)}{R}\right) \mathrm{d} v^{2}-2 \mathrm{~d} R \mathrm{~d} v+R^{2}\left(\mathrm{~d} \theta^{2}+\sin ^{2} \theta \mathrm{d} \phi^{2}\right)
$$

where $v$ is the retarded time and $M(v)$ is the exterior Vaidya mass.

The junction conditions for matching two line elements (3) and (12) continuously across a spherically symmetric time-like hyper surface $\Sigma$ are well known and obtained by (Santos [10])

$$
\begin{aligned}
& (r B)_{\Sigma}=R_{\Sigma}(v)=\mathcal{R}(\tau) \\
& (p)_{\Sigma}=(q B)_{\Sigma} \\
& m_{\Sigma}(r, t)=M(v)=\left\{\frac{r^{3} B \dot{B}^{2}}{2 A^{2}}-r^{2} B^{\prime}-\frac{r^{3} B^{\prime 2}}{2 B}\right\}_{\Sigma}
\end{aligned}
$$

where $m_{\Sigma}$ is the mass function calculated in the interior at $r=r_{\Sigma}$ (Cahill et al. [27] and Misner and Sharp [28]).

Some other characteristics of the model such as the surface luminosity and the boundary redshift observed on $\Sigma$ are

$$
\begin{aligned}
& L_{\Sigma}=\frac{\kappa}{2}\left\{r^{2} B^{3} q\right\}_{\Sigma} \\
& z_{\Sigma}=\left[1+\frac{r B^{\prime}}{B}+\frac{r \dot{B}}{A}\right]_{\Sigma}^{-1}-1
\end{aligned}
$$

The total luminosity for an observer at rest at infinity is

$$
L_{\infty}=-\frac{\mathrm{d} M}{\mathrm{~d} v}=\frac{L_{\Sigma}}{\left(1+Z_{\Sigma}\right)^{2}}
$$

\section{Solution of the Field Equations}

In order to solve the field equations we choose a particular form of the metric coefficients given in (3) into func- 
tions of $r$ and $t$ coordinates as

$$
\begin{aligned}
& A(r, t)=A_{0}(r) g(t) \\
& B(r, t)=B_{0}(r) f(t)
\end{aligned}
$$

In view of (19) and (20) the field Equations (8)-(11) lead to the following system of equations

$$
\begin{aligned}
& \kappa \epsilon=\frac{\epsilon_{0}}{f^{2}}+\frac{3 \dot{f}^{2}}{A_{0}^{2} g^{2} f^{2}} \\
& \kappa p=\frac{p_{0}}{f^{2}}+\frac{1}{A_{0}^{2} g^{2}}\left(-\frac{2 \ddot{f}}{f}-\frac{\dot{f}^{2}}{f^{2}}+\frac{2 \dot{g} \dot{f}}{g f}\right) \\
& \kappa q=-\frac{2 A_{0}^{\prime} \dot{f}}{A_{0}^{2} B_{0}^{2} g f^{3}}
\end{aligned}
$$

where

$$
\begin{aligned}
& \epsilon_{0}=-\frac{1}{B_{0}^{2}}\left(\frac{2 B_{0}^{\prime \prime}}{B_{0}}-\frac{B_{0}^{\prime 2}}{B_{0}^{2}}+\frac{4 B_{0}^{\prime}}{r B_{0}}\right) \\
& p_{0}=\frac{1}{B_{0}^{2}}\left(\frac{B_{0}^{\prime 2}}{B_{0}^{2}}+\frac{2 B_{0}^{\prime}}{r B_{0}}+\frac{2 A_{0}^{\prime} B_{0}^{\prime}}{A_{0} B_{0}}+\frac{2 A_{0}^{\prime}}{r A_{0}}\right)
\end{aligned}
$$

The isotropy of pressure would give the equation

$$
\frac{A_{0}^{\prime \prime}}{A_{0}}+\frac{B_{0}^{\prime \prime}}{B_{0}}=\left(\frac{2 B_{0}^{\prime}}{B_{0}}+\frac{1}{r}\right)\left(\frac{A_{0}^{\prime}}{A_{0}}+\frac{B_{0}^{\prime}}{B_{0}}\right)
$$

In the absence of dissipative force the Equation (14), $\left(p_{r}\right)_{\Sigma}=(q B)_{\Sigma}$, reduces to the condition $\left(p_{0}\right)_{\Sigma}=0$ and yields at $r=r_{\Sigma}=R_{\Sigma}$

$$
\frac{2 \ddot{f}}{f}+\frac{\dot{f}^{2}}{f^{2}}-\frac{2 \dot{g} \dot{f}}{g f}=\frac{2 \alpha g \dot{f}}{f^{2}}
$$

where

$$
\alpha=\left(\frac{A_{0}^{\prime}}{B_{0}}\right)_{\Sigma}
$$

If we assume $g(t)=f(t)$ (Tewari [20]), solution of (26) is

$$
\begin{aligned}
& \dot{f}=2 \alpha f+\beta \sqrt{f} \\
& t=\frac{1}{\alpha} \ln \left(1+\frac{2 \alpha}{\beta} \sqrt{f}\right)
\end{aligned}
$$

where $\beta$ is an arbitrary constant and the constant of integration in (30) has been eliminated by the means of transformation in $t$. The solution (29), (30) is identical to the solution presented by de Oliveira et al. [13], Bonnor et al. [14] with $g(t)=1$. For collapsing configurations we must have $\dot{f}(t) \leq 0$. From Equation (29) we have $\beta \leq-2 \alpha$ as $f(t)$ is positive. We choose $\beta=-2 \alpha$ in order to have $\dot{f} \rightarrow 0$ as $f \rightarrow 1$ the solution (19), (20) and (30) represents a static perfect fluid at $t \rightarrow-\infty$ and then the fluid gradually starts evolving into a non-adiabatic radiating collapse.

Equations (29) and (30) become

$$
\begin{aligned}
& \dot{f}=-2 \alpha \sqrt{f}(1-\sqrt{f}) \\
& t=\frac{1}{\alpha} \ln (1-\sqrt{f})
\end{aligned}
$$


We observed that the function $f(t)$ decreases monotonically from the value $f(t)=1$ at $t=-\infty$ to $f(t)=0$ at $t=0$.

The new parametric class of solutions of Equation (26) obtained by Tewari [20] is given as

$$
\begin{aligned}
& A_{0}=D_{2}\left(1+C_{1} r^{2}\right)^{\frac{n}{l+1}}+D_{1}\left(1+C_{1} r^{2}\right)^{\frac{2-n}{l+1}+1} \\
& B_{0}=C_{2}\left(1+C_{1} r^{2}\right)^{\frac{1}{l+1}}
\end{aligned}
$$

where $n, l, C_{1}, C_{2}, D_{1}$ and $D_{2}$ are constants and

$$
n=\frac{1}{2}\left\{(l+3) \pm\left(l^{2}+10 l+17\right)^{\frac{1}{2}}\right\}
$$

$n$ is real if $l \geq-5+2 \sqrt{2}$ or $l \leq-5-2 \sqrt{2}$.

For different values of $n$ or $l$ Equations (33) and (34) give a variety of solutions (in Table 1). For $n=0,1$; we rediscover the Schwarzschild interior solution and the collapsing radiating star model in this case has been studied by de Oliveira et al. [13] and Bonnor et al. [14] and for $n=-1$, the solution reduces to Banerjee et al. [15], for $n=-2,-\frac{3}{2}$, it reduces to Tewari [19] [20], for $n=-1-\sqrt{2}$, horizon-free case studied by Tewari and Cha-

\begin{tabular}{|c|c|c|c|c|}
\hline s.n. & $n$ & $l$ & $A_{0}$ & $B_{0}$ \\
\hline 1 & $\frac{5 \pm \sqrt{41}}{2}$ & 2 & $D_{2}\left(1+C_{1} r^{2}\right)^{\frac{5+\sqrt{41}}{6}}+D_{1}\left(1+C_{1} r^{2}\right)^{\frac{5-\sqrt{41}}{6}}$ & $C_{2}\left(1+C_{1} r^{2}\right)^{\frac{1}{3}}$ \\
\hline 2 & $2 \pm \sqrt{7}$ & 1 & $D_{2}\left(1+C_{1} r^{2}\right)^{\frac{2+\sqrt{7}}{2}}+D_{1}\left(1+C_{1} r^{2}\right)^{\frac{2-\sqrt{7}}{2}}$ & $C_{2}\left(1+C_{1} r^{2}\right)^{\frac{1}{2}}$ \\
\hline 3 & $\frac{3 \pm \sqrt{17}}{2}$ & 0 & $D_{2}\left(1+C_{1} r^{2}\right)^{3+\sqrt{17}}+D_{1}\left(1+C_{1} r^{2}\right)^{\frac{3-\sqrt{17}}{2}}$ & $C_{2}\left(1+C_{1} r^{2}\right)$ \\
\hline 4 & $-1+\sqrt{2}$ & $-5+2 \sqrt{2}$ & $\left(D_{2}+D_{1}\right)\left(1+C_{1} r^{2}\right)^{\frac{-\sqrt{2}}{4}}$ & $C_{2}\left(1+C_{1} r^{2}\right)^{\frac{-(2+\sqrt{2})}{4}}$ \\
\hline 5 & 1,0 & -2 & $D_{2}+D_{1}\left(1+C_{1} r^{2}\right)^{-1}$ & $C_{2}\left(1+C_{1} r^{2}\right)^{-1}$ \\
\hline 6 & $-1-\sqrt{2}$ & $-5-2 \sqrt{2}$ & $\left(D_{2}+D_{1}\right)\left(1+C_{1} r^{2}\right)^{\frac{\sqrt{2}}{4}}$ & $C_{2}\left(1+C_{1} r^{2}\right)^{\frac{-2+\sqrt{2}}{4}}$ \\
\hline 7 & $-3,-2$ & -8 & $D_{2}\left(1+C_{1} r^{2}\right)^{\frac{2}{7}}+D_{1}\left(1+C_{1} r^{2}\right)^{\frac{3}{7}}$ & $C_{2}\left(1+C_{1} r^{2}\right)^{\frac{-1}{7}}$ \\
\hline 8 & $-4,-5 / 3$ & $-26 / 3$ & $D_{2}\left(1+C_{1} r^{2}\right)^{\frac{5}{23}}+D_{1}\left(1+C_{1} r^{2}\right)^{\frac{12}{23}}$ & $C_{2}\left(1+C_{1} r^{2}\right)^{\frac{-3}{23}}$ \\
\hline 9 & $-5,-3 / 2$ & $-19 / 2$ & $D_{2}\left(1+C_{1} r^{2}\right)^{\frac{3}{17}}+D_{1}\left(1+C_{1} r^{2}\right)^{\frac{10}{17}}$ & $C_{2}\left(1+C_{1} r^{2}\right)^{\frac{-2}{17}}$ \\
\hline 10 & $-6,-7 / 5$ & $-52 / 5$ & $D_{2}\left(1+C_{1} r^{2}\right)^{\frac{7}{47}}+D_{1}\left(1+C_{1} r^{2}\right)^{\frac{30}{47}}$ & $C_{2}\left(1+C_{1} r^{2}\right)^{\frac{-5}{47}}$ \\
\hline 11 & $-7,-4 / 3$ & $-34 / 3$ & $D_{2}\left(1+C_{1} r^{2}\right)^{\frac{4}{31}}+D_{1}\left(1+C_{1} r^{2}\right)^{\frac{21}{31}}$ & $C_{2}\left(1+C_{1} r^{2}\right)^{\frac{-3}{31}}$ \\
\hline 12 & $-8,-9 / 7$ & $-86 / 7$ & $D_{2}\left(1+C_{1} r^{2}\right)^{\frac{9}{99}}+D_{1}\left(1+C_{1} r^{2}\right)^{\frac{56}{9}}$ & $C_{2}\left(1+C_{1} r^{2}\right)^{\frac{-7}{79}}$ \\
\hline 13 & $-9,-5 / 4$ & $-53 / 4$ & $D_{2}\left(1+C_{1} r^{2}\right)^{\frac{5}{49}}+D_{1}\left(1+C_{1} r^{2}\right)^{\frac{366}{49}}$ & $C_{2}\left(1+C_{1} r^{2}\right)^{\frac{-4}{49}}$ \\
\hline 14 & $-10,-11 / 9$ & $-128 / 9$ & $D_{2}\left(1+C_{1} r^{2}\right)^{\frac{11}{119}}+D_{1}\left(1+C_{1} r^{2}\right)^{\frac{90}{119}}$ & $C_{2}\left(1+C_{1} r^{2}\right)^{\frac{-9}{119}}$ \\
\hline 15 & $-11,-6 / 5$ & $-76 / 5$ & $D_{2}\left(1+C_{1} r^{2}\right)^{\frac{6}{11}}+D_{1}\left(1+C_{1} r^{2}\right)^{\frac{55}{11}}$ & $C_{2}\left(1+C_{1} r^{2}\right)^{\frac{-5}{11}}$ \\
\hline
\end{tabular}
$\operatorname{ran}[21]$. We here present some more special solutions and a detailed study of a class of solutions.

\section{Table 1. Different values of $n$ or $l$.}


4. Detailed Study of a New Class of Exact Solutions Corresponding to $n=-\frac{5}{3}$ for a

\section{Collapsing Radiating Star}

In order to construct the new realistic model we assume $n=-\frac{5}{3}$, and from (33) and (34) we obtain

$$
\begin{aligned}
& A_{0}=D_{2}\left(1+C_{1} r^{2}\right)^{\frac{5}{23}}+D_{1}\left(1+C_{1} r^{2}\right)^{\frac{12}{23}} \\
& B_{0}=C_{2}\left(1+C_{1} r^{2}\right)^{\frac{-3}{23}}
\end{aligned}
$$

In view of (36) and (37) we obtain from (24) and (25)

$$
\begin{gathered}
\epsilon_{0}=\frac{12 C_{1}}{529 C_{2}^{2}\left(1+C_{1} r^{2}\right)^{\frac{40}{23}}}\left(69+20 C_{1} r^{2}\right) \\
p_{0}=\frac{4 C_{1}}{529 C_{2}^{2}\left(1+C_{1} r^{2}\right)^{\frac{40}{23}}}\left[\left(46+25 C_{1} r^{2}\right)+\frac{7 D_{1}\left(1+C_{1} r^{2}\right)^{\frac{7}{23}}\left(23+17 C_{1} r^{2}\right)}{\left\{D_{2}+D_{1}\left(1+C_{1} r^{2}\right)^{\frac{7}{23}}\right\}}\right]
\end{gathered}
$$

The junction condition $\left(p_{0}\right)_{\Sigma}=0$ gives

$$
D_{2}=\frac{-9 D_{1}\left(1+C_{1} r_{\Sigma}^{2}\right)^{\frac{7}{23}}\left(23+16 C_{1} r_{\Sigma}^{2}\right)}{\left(46+25 C_{1} r_{\Sigma}^{2}\right)}
$$

The central values of $\epsilon_{0}$ and $p_{0}$ are given by

$$
\begin{aligned}
& \left(\epsilon_{0}\right)_{r=0}=\frac{36 C_{1}}{23 C_{2}^{2}} \\
& \left(p_{0}\right)_{r=0}=\frac{4 C_{1}}{23 C_{2}^{2}}\left[2+\frac{7 D_{1}}{D_{2}+D_{1}}\right]
\end{aligned}
$$

A physically reasonable solution should satisfy certain conditions. They are

(i) The central values of pressure, density and metric potential component should be non-zero positive definite.

This condition is satisfied if $C_{1}>0, C_{2}>0, D_{2}>\frac{-9}{2} D_{1}$ and subjecting to the condition $\frac{p_{0}}{\epsilon_{0}}<1$, yields $D_{2}>0$, which holds good if $D_{1}<0$.

(ii) The solution should have monotonically decreasing expressions for the pressure and density with respect to $r$. For this, using (38) and (39) respectively we get

$$
\epsilon_{0}^{\prime}=\frac{-480 C_{1}^{2} r}{12167 C_{2}^{2}}\left[\frac{115+17 C_{1} r^{2}}{\left(1+C_{1} r^{2}\right)^{\frac{63}{23}}}\right]
$$

Thus the extrema of $\epsilon_{0}$ occurs at the centre if $\epsilon_{0}^{\prime}=0 \Rightarrow r=0$,

$$
\left(\epsilon_{0}^{\prime \prime}\right)_{r=0}=\frac{-2400 C_{1}^{2}}{529 C_{2}^{2}}<0, \text { holds for all the values of } C_{1} \text { and } C_{2}
$$




$$
p_{0}^{\prime}=\frac{-8 C_{1}^{2} r}{12167 C_{2}^{2}\left(1+C_{1} r^{2}\right)^{\frac{56}{23}}}\left[\frac{\left(1265+425 C_{1} r^{2}\right)}{\left(1+C_{1} r^{2}\right)^{\frac{5}{23}}}+\frac{7 D_{1}\left\{D_{2}\left(368+170 C_{1} r^{2}\right)+D_{1}\left(1+C_{1} r^{2}\right)^{\frac{7}{23}}\left(529+289 C_{1} r^{2}\right)\right\}}{\left\{D_{2}+D_{1}\left(1+C_{1} r^{2}\right)^{\frac{7}{23}}\right\}^{2}}\right]
$$

Thus the extrema of $p_{0}$ occurs at the centre if $p_{0}^{\prime}=0 \Rightarrow r=0$,

$$
\left(p_{0}^{\prime \prime}\right)_{r=0}=\frac{-8 C_{1}^{2}}{12167 C_{2}^{2}}\left[\frac{4968 D_{1}^{2}+1265 D_{2}^{2}+5106 D_{1} D_{2}}{\left(D_{2}+D_{1}\right)^{2}}\right]<0 \text {, holds for } D_{2}>\frac{-12}{5} D_{1}
$$

The above observed range of $D_{2}$ is covered under energy condition (i).

Thus the expressions of right hand side of (44) and (46) are hold good for all the already mentioned ranges of parameters satisfying physical viability of the model, showing thereby that the density and pressure are maximum at the centre and monotonically decreasing towards the boundary surface.

Further, it is mentioned here that the boundary of the collapsing radiating star is established only when $C_{1}>0, C_{2}>0, D_{2}>\frac{-9}{2} D_{1}$ and $D_{1}<0$.

By using (7), (21) - (23), (31), (32), (36) and (37) the explicit expressions for $\epsilon, p, q$ and $\Theta$ become

$$
\begin{aligned}
& \kappa \epsilon=\frac{\epsilon_{0}}{f^{2}}+\frac{12 \alpha^{2}}{A_{0}^{2}} \frac{(1-\sqrt{f})^{2}}{f^{3}} \\
& \kappa p=\frac{p_{0}}{f^{2}}+\frac{4 \alpha^{2}}{A_{0}^{2}} \frac{(1-\sqrt{f})}{f^{\frac{5}{2}}} \\
& \kappa q=\frac{4 C_{1} r\left\{\frac{5}{23} D_{2}+\frac{12}{23} D_{1}\left(1+C_{1} r^{2}\right)^{\frac{7}{23}}\right\}}{C_{2}^{2}\left(1+C_{1} r^{2}\right)^{\frac{22}{23}}\left\{D_{2}+D_{1}\left(1+C_{1} r^{2}\right)^{\frac{7}{23}}\right\}^{2}} \frac{2 \alpha(1-\sqrt{f})}{f^{\frac{7}{2}}} \\
& \Theta=\frac{-6 \alpha(1-\sqrt{f})}{\left(1+C_{1} r^{2}\right)^{\frac{5}{23}}\left\{D_{2}+D_{1}\left(1+C_{1} r^{2}\right)^{\frac{7}{23}}\right\} f^{\frac{3}{2}}}
\end{aligned}
$$

where

$$
\alpha=\frac{-42}{23} \frac{C_{1} D_{1} r_{\Sigma}}{C_{2}\left(1+C_{1} r_{\Sigma}^{2}\right)^{\frac{8}{23}}}\left[\frac{\left(23+20 C_{1} r_{\Sigma}^{2}\right)}{\left(46+25 C_{1} r_{\Sigma}^{2}\right)}\right]
$$

We can see the physical parameters $\epsilon, p, q$ are finite, positive, monotonically decreasing at any instant with respect to radial coordinate for $0 \leq r \leq r_{\Sigma}$. Initially collapse is zero and it becomes infinite at the final phase of the configuration. The constant $\alpha$ is positive for the given range of the parameters $C_{1}, D_{1}$ and $C_{2}$.

The total energy entrapped inside $\Sigma$ is given by (15), which becomes, using (19), (20), (31), (32), (36) and (37)

$$
M(v)=\left[2\left(\frac{6}{23}\right)^{2} \cdot \frac{C_{2} C_{1}^{2} r_{\Sigma}^{5}}{\left(1+C_{1} r_{\Sigma}^{2}\right)^{\frac{49}{23}}} \cdot \frac{\left(23+20 C_{1} r_{\Sigma}^{2}\right)^{2}}{\left(23+17 C_{1} r_{\Sigma}^{2}\right)^{2}}(1-\sqrt{f})^{2}+m_{0} f\right]_{\Sigma}
$$

where 


$$
m_{0}=\frac{6 C_{1} C_{2} r_{\Sigma}^{3}\left(23+20 C_{1} r_{\Sigma}^{2}\right)}{529\left(1+C_{1} r_{\Sigma}^{2}\right)^{\frac{49}{23}}}
$$

Using (16) - (20), (31), (32), (36) and (37) the luminosity and the red shift observed on $\Sigma$ and luminosity observed by a distant observer are given by

$$
\begin{aligned}
& L_{\Sigma}=2\left(\frac{6}{23}\right)^{2} \cdot \frac{C_{1}^{2} r_{\Sigma}^{4}}{\left(1+C_{1} r_{\Sigma}^{2}\right)^{2}} \cdot \frac{\left(23+20 C_{1} r_{\Sigma}^{2}\right)^{2}}{\left(23+17 C_{1} r_{\Sigma}^{2}\right)^{2}} \cdot \frac{(1-\sqrt{f})}{\sqrt{f}} \\
& L_{\infty}=2\left(\frac{6}{23}\right)^{2} \cdot \frac{C_{1}^{2} r_{\Sigma}^{4}}{\left(1+C_{1} r_{\Sigma}^{2}\right)^{2}} \cdot \frac{\left(23+20 C_{1} r_{\Sigma}^{2}\right)^{2}}{\left(23+17 C_{1} r_{\Sigma}^{2}\right)^{2}} \cdot \frac{(1-\sqrt{f})}{\sqrt{f}} \cdot \frac{1}{\left(1+Z_{\Sigma}\right)^{2}} \\
& z_{\Sigma}=\left[1-\frac{6 C_{1} r_{\Sigma}^{2}}{23\left(1+C_{1} r_{\Sigma}^{2}\right)}-\frac{12 C_{1} r_{\Sigma}^{2}\left(23+20 C_{1} r_{\Sigma}^{2}\right)}{23\left(1+C_{1} r_{\Sigma}^{2}\right)\left(23+17 C_{1} r_{\Sigma}^{2}\right)} \frac{(1-\sqrt{f})}{\sqrt{f}}\right]_{\Sigma}^{-1}-1
\end{aligned}
$$

The Equations (55) and (56) show that $L_{\infty}$ vanishes in the beginning when $f(t) \rightarrow 1$ and at the stage when $z_{\Sigma} \rightarrow \infty$.

The black hole formation time when the collapse reaches the horizon of the black hole occurs when the surface redshift goes to infinity, for this the term in the parentheses in Equation (56) goes to zero and we obtain

$$
\sqrt{f_{B H}}=\frac{12 C_{1} r_{\Sigma}^{2}\left(23+20 C_{1} r_{\Sigma}^{2}\right)}{529\left(1+C_{1} r_{\Sigma}^{2}\right)^{2}}
$$

and using (32) in view of (57) we have

$$
t_{B H}=\frac{1}{\alpha} \ln \frac{\left(23+17 C_{1} r_{\Sigma}^{2}\right)^{2}}{529\left(1+C_{1} r_{\Sigma}^{2}\right)^{2}}
$$

\section{Temperature Profile}

Now we will investigate the evolution of temperature of the collapsing star. In view of extended irreversible thermodynamics, the relativistic Maxwell-Cattaneo relation for temperature governing the heat transport within the collapsing matter in the truncated Israel-Stewart theory has the form (Israel et al. [29], Maartens [30], and Martinez [31])

$$
\tau\left(g^{\mu v}+w^{\mu} w^{v}\right) w^{\alpha} q_{v ; \alpha}+q^{\mu}=-\mathbb{K}\left(g^{\mu v}+w^{\mu} w^{v}\right)\left[T_{, v}+T \dot{w}_{v}\right]
$$

where $\mathbb{K}(\geq 0)$ is the thermal conductivity and $\tau(\geq 0)$ is the relaxation time. To get a simple estimate of the temperature evolution, by setting $\tau=0$ in (59) we get

$$
q=-\mathbb{K} \frac{1}{B_{0}^{2} f^{2}}\left(T^{\prime}+T \frac{A_{0}^{\prime}}{A_{0}}\right)=-\frac{2 A_{0}^{\prime} \dot{f}}{A_{0}^{2} B_{0}^{2} f^{4}}
$$

If we assume thermal conductivity $\mathbb{K}=\gamma T^{\Omega} \geq 0$, where $\gamma$ and $\Omega$ are positive constants, on integration (60) yields

$$
T^{\Omega+1}=\frac{T_{0}(t)}{A_{0}^{\Omega+1}}-\frac{4(\Omega+1)}{\gamma \Omega} \frac{\alpha}{A_{0}} \frac{(1-\sqrt{f})}{f^{\frac{3}{2}}}
$$

where $T_{0}(t)$ is an arbitrary function of $t$.

The effective surface temperature observed by external observer can be calculated from the expression 
(Schwarzschild [32])

$$
T_{\Sigma}^{4}=\left\{\frac{1}{\pi \delta\left(r B_{0} f\right)^{2}}\right\}_{\Sigma} L_{\infty}=\frac{72}{529 \pi \delta C_{2}^{2}} \frac{C_{1}^{2} r_{\Sigma}^{2}\left(23+20 C_{1} r_{\Sigma}^{2}\right)^{2}}{\left(1+C_{1} r_{\Sigma}^{2}\right)^{\frac{40}{23}}\left(23+17 C_{1} r_{\Sigma}^{2}\right)^{2}} \frac{(1-\sqrt{f})}{f^{\frac{5}{2}}} \frac{1}{\left(1+Z_{\Sigma}\right)^{2}}
$$

The effective surface temperature is zero in the beginning $(f(t) \rightarrow 1)$ and at the stage when collapsing configuration reaches the horizon of the black hole $\left(z_{\Sigma} \rightarrow \infty\right)$.

where the constant $\delta$ in Photon is given by

$$
\delta=\frac{\pi^{2} k^{4}}{15 \hbar^{3}}
$$

where $k$ and $\hbar$ denoting respectively Boltzmann and Plank constants.

Choosing $\Omega=3$ which represents radiation interaction with matter through the diffusive approximation (Misner and Sharp [33]). The arbitrary function $T_{0}(t)$ is determined by using (61) and (62) as

$$
\begin{aligned}
T_{0}(t)= & \left\{\frac{16 \alpha}{3 k \gamma} \frac{\left[D_{2}+D_{1}\left(1+C_{1} r^{2}\right)^{\frac{7}{23}}\right]^{3}\left(1+C_{1} r^{2}\right)^{\frac{15}{23}}(1-\sqrt{f})}{f^{\frac{3}{2}}}\right\}_{\Sigma} \\
& +\left\{\frac{2 \alpha^{2}\left[D_{2}+D_{1}\left(1+C_{1} r^{2}\right)^{\frac{7}{23}}\right]^{2}\left(1+C_{1} r^{2}\right)^{\frac{10}{23}}}{\pi \delta r^{2}} \frac{(1-\sqrt{f})}{f^{\frac{5}{2}}}\right\}_{\Sigma} \frac{1}{\left(1+Z_{\Sigma}\right)^{2}}
\end{aligned}
$$

The temperature inside the star is given by

$$
T^{4}=\left[\frac{T_{0}(t)}{\left[D_{2}+D_{1}\left(1+C_{1} r^{2}\right)^{\frac{7}{23}}\right]^{4}\left(1+C_{1} r^{2}\right)^{\frac{20}{23}}}-\frac{16 \alpha}{3 \gamma\left(1+C_{1} r^{2}\right)^{\frac{5}{23}}\left[D_{2}+D_{1}\left(1+C_{1} r^{2}\right)^{\frac{7}{23}}\right.} \frac{(1-\sqrt{f})}{f^{\frac{3}{2}}}\right]
$$

\section{Conclusion}

We have proposed some special solutions of Tewari [20] and a new class of exact solutions corresponding to $n=-\frac{5}{3}$ has been studied in detail. The interior metric is in separable form and the model seems to be physically and thermodynamically sound as it corresponds to well-behaved nature for fluid density, isotropic pressure and radiation flux density throughout the fluid sphere. Initially the interior solutions represent a static configuration of non-dissipative fluid which then gradually starts evolving into radiating collapse. The apparent luminosity as observed by the distant observer at rest at infinity and the effective surface temperature are zero in remote past at the instant when the collapse begins and at the stage when collapsing configuration reaches the horizon of the black hole.

\section{Acknowledgements}

We thank the anonymous referee for valuable suggestions.

\section{References}

[1] Penrose, R. (1969) Rivista Del Nuovo Cimento, 1, 252-276. 
[2] Joshi, P.S. and Malafarina, D. (2011) International Journal of Modern Physics D, 20, 2641-2729. http://dx.doi.org/10.1142/S0218271811020792

[3] Oppenheimer, J.R. and Snyder, H. (1939) Physical Review, 56, 455-459. http://dx.doi.org/10.1103/PhysRev.56.455

[4] Vaidya, P.C. (1951) Proceedings of the Indian Academy of Sciences-Section A, 33, 264-276.

[5] Misner, C.W. (1965) Physical Review B, 137, 1360-1364. http://dx.doi.org/10.1103/PhysRev.137.B1360

[6] Lindquist, R.W., Schwartz, R.A. and Misner, C.W. (1965) Physical Review B, 137, 1364-1368. http://dx.doi.org/10.1103/PhysRev.137.B1364

[7] Herrera, L. and Santos, N.O. (2004) Physical Review D, 70, Article ID: 084004. http://dx.doi.org/10.1103/PhysRevD.70.084004

[8] Herrera, L., Di Prisco, A., Martin, J. and Ospino, J. (2006) Physical Review D, 74, Article ID: 044001. http://dx.doi.org/10.1103/PhysRevD.74.044001

[9] Mitra, A. (2006) Physical Review D, 74, 024010. http://dx.doi.org/10.1103/PhysRevD.74.024010

[10] Santos, N.O. (1985) Monthly Notices of the Royal Astronomical Society, 216, 403-410.

[11] Glass, E.N. (1981) Physics Letters A, 86, 351-352. http://dx.doi.org/10.1016/0375-9601(81)90553-3

[12] Maiti, S.R. (1982) Physical Review D, 25, 2518-2521. http://dx.doi.org/10.1103/PhysRevD.25.2518

[13] de Oliveira, A.K.G., Santos, N.O. and Kolassis, C.A. (1985) Monthly Notices of the Royal Astronomical Society, 216, 1001-1011. http://dx.doi.org/10.1093/mnras/216.4.1001

[14] Bonnor, W.B., de Oliveira, A.K.G. and Santos, N.O. (1989) Physics Reports, 181, 269-326. http://dx.doi.org/10.1016/0370-1573(89)90069-0

[15] Banerjee, A., Chaterjee, S. and Dadhich, N. (2002) Modern Physics Letters A, 17, 2335-2339. http://dx.doi.org/10.1142/S0217732302008320

[16] Herrera, L., Di Prisco, A., Ospino, J., Fuenmayor, E. and Triconis, O. (2009) Physical Review D, 79, Article ID: 064025. http://dx.doi.org/10.1103/PhysRevD.79.064025

[17] Tewari, B.C. (1988) Astrophysics and Space Science, 149, 233-239. http://dx.doi.org/10.1007/BF00639793

[18] Tewari, B.C. (2010) Radiating Fluid Balls in General Relativity. VDM Verlag, Saarbrucken.

[19] Tewari, B.C. (2012) Astrophysics and Space Science, 342, 73-77. http://dx.doi.org/10.1007/s10509-012-1141-3

[20] Tewari, B.C. (2013) General Relativity and Gravitation, 45, 1547-1558. http://dx.doi.org/10.1007/s10714-013-1545-6

[21] Tewari, B.C. and Charan, K. (2014) Astrophysics and Space Science, 351, 613-617. http://dx.doi.org/10.1007/s10509-014-1851-9

[22] Sharma, R. and Tikekar, R. (2012) General Relativity and Gravitation, 44, 2503-2520. http://dx.doi.org/10.1007/s10714-012-1406-8

[23] Ivanov, B.V. (2012) General Relativity and Gravitation, 44, 1835-1855. http://dx.doi.org/10.1007/s10714-012-1370-3

[24] Pinheiro, G. and Chan, R. (2013) General Relativity and Gravitation, 45, 243-261. http://dx.doi.org/10.1007/s10714-012-1468-7

[25] Vaidya, P.C. (1953) Nature, 171, 260-261. http://dx.doi.org/10.1038/171260a0

[26] Glass, E.N. (1979) Journal of Mathematical Physics, 20, 1508-1513. http://dx.doi.org/10.1063/1.524210

[27] Cahill, M.E. and McVittie, G.C. (1970) Journal of Mathematical Physics, 11, 1382-1391. http://dx.doi.org/10.1063/1.1665273

[28] Misner, C.W. and Sharp, D.H. (1964) Physical Review, 136, 571-576. http://dx.doi.org/10.1103/PhysRev.136.B571

[29] Israel, W. and Stewart, J. (1979) Annals of Physics, 118, 341-372. http://dx.doi.org/10.1016/0003-4916(79)90130-1

[30] Maartens, R. (1995) Classical and Quantum Gravity, 12, 1455-1465. http://dx.doi.org/10.1088/0264-9381/12/6/011

[31] Martinez, J. (1996) Physical Review D, 53, 6921-6940. http://dx.doi.org/10.1103/PhysRevD.53.6921

[32] Schwarzschild, M. (1958) Structure and Evolution of Stars. Dover, New York.

[33] Misner, C.W. and Sharp, D.H. (1965) Physics Letters, 15, 279-281. http://dx.doi.org/10.1016/0031-9163(65)91247-3 